\title{
ATRIBUTOS FUNCIONAIS QUE PODEM CONTRIBUIR COM O DESENVOLVIMENTO DA MODELAGEM DO MOODLE: ENSINO PRESENCIAL DO IFC - CAMPUS CAMBORIÚ
}

\author{
Fernanda Borges Vaz Ribeiro, IFC-CAMBORIÚ, fernanda.ribeiro@ifc.edu.br \\ Marilda Todescat, CAD/UFSC, marildat@gmail.com
}

\begin{abstract}
Resumo: $\mathrm{O}$ artigo tem como objetivo propor atributos funcionais por meio da análise das categorias usabilidade, interação, colaboração e aprendizagem a fim de contribuir com a modelagem do ambiente virtual de aprendizagem (AVA) Moodle para o ensino presencial do IFC - Campus Camboriú. A abordagem deste estudo é qualitativa. Quanto aos fins, a pesquisa é descritiva e quanto aos meios, bibliográfica e documental e se enquadra em um estudo de caso. Como resultados deste estudo, constataram-se as ferramentas consideradas essenciais pelos professores, tutores e discentes do Curso de Pós-Graduação à distância (EAD) em Educação Básica Integrada e Educação Profissional na modalidade de Jovens e Adultos (PROEJA), do IFC Campus Camboriú; pontos fortes e fracos da plataforma Moodle e por fim, foram detectados atributos funcionais que podem favorecer a modelagem do AVA Moodle para o ensino presencial do IFC - Campus Camboriú.
\end{abstract}

Palavras-Chave: Ambientes Virtuais de Aprendizagem. Avaliação. Moodle.

\section{FUNCTIONAL ATTRIBUTES THAT COULD CONTRIBUTE TO THE DEVELOPMENT OF MOODLE MODELING: IFC - CAMPUS CAMBORIÚ PRESENT TEACHING}

\begin{abstract}
The article aims to propose functional attributes through the analysis of the categories usability, interaction, collaboration and learning in order to contribute to the modeling of the Virtual Learning Environment (AVA) Moodle for the classroom teaching of IFC - Campus Camboriu. The approach of this study is qualitative. As for the purposes, the research is descriptive and as to the media, bibliographic and documentary and it falls into a case study. As a result of this study, the tools considered essential by the teachers, tutors and students of the distance Graduate Course (EAD) in integrated basic education and professional education in the modality of youth and adult (protect), IFC Campus Camboriú; Strengths and weaknesses of the Moodle platform and finally, functional attributes have been detected that may favor the modeling of AVA Moodle for the teaching of IFC - Campus Camboriu
\end{abstract}

Keywords: Virtual learning environments. Evaluation. Moodle.

\section{INTRODUÇÃO}

O artigo é fruto dos resultados da pesquisa de mestrado intitulada "atributos funcionais que podem contribuir com o desenvolvimento da modelagem do Moodle: ensino presencial do IFC - Campus Camboriú".

A pesquisa foi realizada no $2^{\circ}$ semestre de 2014, envolvendo a infraestrutura do curso de Pós-Graduação EAD em PROEJA do IFC Campus Camboriú, sendo parte dela constituída pelo Coordenador de curso; 5 (cinco) professores; 4 (quatro) tutores; 1 (um) coordenador de suporte à EAD; 3 (três) profissionais de Tecnologia da Informação (TI) e 29 (vinte e nove) discentes concluintes do Curso de Pós-Graduação EAD PROEJA. Também fizeram parte desta pesquisa 2 (dois) profissionais de TI da Superintendência de 
Governança Eletrônica e Tecnologia da Informação e Comunicação (SETIC) pertencentes à Universidade Federal de Santa Catarina (UFSC).

O estudo foi baseado na análise das categorias usabilidade, interação, colaboração e aprendizagem, a partir do modelo de avaliação de AVAs interacionista/construtivista proposto por Schlemmer e Fagundes (2001) que consideram as perspectivas técnica, comunicacional-social, pedagógica e de gestão. Estas perspectivas apresentam o objetivo de fomentar a melhoria da qualidade da aprendizagem, dar suporte aos processos comunicacionais e reduzir a sobrecarga administrativa dos docentes por meio de um gerenciamento mais ágil, permitindo-lhes maior dedicação às demandas dos discentes.

$\mathrm{O}$ objetivo da pesquisa foi identificar atributos funcionais quanto à usabilidade, interação, colaboração e aprendizagem que podem colaborar com a modelagem do AVA Moodle para o ensino presencial do IFC - Campus Camboriú, a partir da análise das funcionalidades do Moodle utilizado pelos Profissionais e discentes do Curso de PósGraduação EAD PROEJA do IFC Campus Camboriú.

Este artigo está dividido em cinco seções. Na primeira apresenta-se a introdução. Na segunda, o referencial teórico sobre Parâmetros para avaliação de AVAs. Na terceira, a metodologia da pesquisa. $\mathrm{Na}$ quarta são propostos atributos funcionais que podem contribuir com a modelagem do AVA Moodle no ensino presencial do IFC - Campus Camboriú. E na última seção as considerações finais.

\section{PARÂMETROS PARA AVALIAÇÃO DE AVAS}

A educação vivencia um momento em que a combinação de pequenos ajustes nas formas culturais de aprender e ensinar não é mais suficiente para enfrentar os desafios das novas gerações que se encontram imersas em uma nova realidade tecnológica. Sendo assim, é necessário fazer uma mudança profunda nas estruturas e hábitos dos antigos métodos educacionais (COLL; MONEREO, 2010).

Um novo espaço é oportunizado pelas tecnologias da informação e da comunicação com o surgimento dos AVAs. Para Haguenauer, Lima e Cordeiro Filho (2010), o AVA é uma ferramenta com uma perspectiva pedagógica na qual o docente tem a possibilidade de disponibilizar diversos recursos para o aluno, bem como, textos, aulas, cronogramas e exercícios, entre outros. Nesse sentido, a comunicação pode ser fomentada de modo síncrono (em tempo real) ou assíncrono, quando alunos, professores e colegas de classe podem efetuar a comunicação em tempos distintos, como blogs ou mural virtual.

A estruturação de uma atividade ou um curso em um AVA necessita de um estudo prévio levando em consideração diversos fatores. Do mesmo modo, quando se pretende identificar atributos funcionais que podem contribuir com a modelagem de um ambiente virtual de aprendizagem, existem critérios, parâmetros e metodologias de avaliação que auxiliam a equipe multidisciplinar na escolha de recursos e tecnologias adequadas de modo a garantir a qualidade e a eficácia do processo de aprendizagem em um AVA.

A avaliação de AVAs estabelece metodologias por meio do estudo e análise das ferramentas e recursos disponibilizados. Ademais, propicia benefícios, facilidades e oportunidades aos participantes desses ambientes, incluindo suas especificações técnicas e o custo. Além disso, os instrumentos de avaliação de AVAs são essenciais e dão um feedback aos desenvolvedores e educadores sobre a multiplicidade de aspectos presentes no ambiente, tais como, sua usabilidade, ergonomia, confiabilidade, acessibilidade, interação e do ponto de vista pedagógico (SCHLEMMER; SACCOL; GARRIDO, 2006, 2007; OLIVEIRA, 2001).

Partindo da premissa que o AVA Moodle é baseado na teoria construtivista, optou-se por analisar o modelo de avaliação interacionista/construtivista estruturado por 
Schlemmer e Fagundes (2002), a partir das perspectivas técnica, didático-pedagógica, comunicacional-social e administrativa que considera, também, a sua aplicação por equipes interdisciplinares constituídas por usuários, educadores, pedagogos, equipe de TI e gestores.

De acordo com Schlemmer e Fagundes (2001) três ações devem ser aplicadas numa estratégia de avaliação de AVAs, a saber:

a) fomentar a melhoria da qualidade da aprendizagem, utilizando-se de métodos não convencionais;

b) dar suporte à processos comunicacionais que possibilitem um alto nível de interatividade fortalecendo o trabalho em equipe;

c) reduzir a sobrecarga administrativa dos professores possibilitando que façam o gerenciamento mais ágil de sua carga de trabalho, permitindo-lhes maior dedicação às necessidades educacionais individuais dos discentes.

Nesse contexto, apresenta-se uma breve conceituação das categorias propostas por Schlemmer e Fagundes (2001): técnica, comunicacional-social, administrativa e didático pedagógica.

\subsection{Perspectivas Técnica, Comunicacional-social, Administrativa e Didádico- pedagógica}

A Técnica analisa o suporte tecnológico que o sistema deve apresentar, incluindo ferramentas que permitem o gerenciamento do ambiente, tal como, suporte tecnológico para as ferramentas disponibilizadas pelo AVA (ferramentas de autoria, de trabalho individual e coletivo e serviços diversos) (SCHLEMMER; FAGUNDES, 2001; SCHLEMMER, 2005).

A Comunicacional-social conforme a abordagem construtivista-social tem sua essência na comunicação, posto que é necessário que a interação aconteça entre os usuários envolvidos no processo de aprendizagem, oportunizando a interação e a construção colaborativa de possibilidades dentro do ambiente conduzindo à construção do conhecimento de maneira cooperativa (SCHLEMMER; FAGUNDES, 2001; SCHLEMMER, 2005).

A Administrativa considera questões condizentes à administração dos usuários dentro do AVA e o papel dos diferentes atores a partir da análise dos seguintes enfoques administrativos:

a) Adaptabilidade - possibilita ao professor adaptar as atividades de acordo com as necessidades individuais ou em grupos;

b) Monitoramento - possibilita que o professor monitore se a aprendizagem está ocorrendo e como está ocorrendo;

c) Organização - permite a criação de grupos para a organização dos alunos sem requerer alguma ação do professor;

d) Estrutura - em rede, interdisciplinar. A organização se dá por áreas, células, disciplinas e temas (SCHLEMMER; FAGUNDES, 2001; SCHLEMMER, 2005).

A Didático-pedagógica analisa questões epistemológicas e paradigmas educacionais que fundamentam a criação de um AVA. Entretanto, quando o contexto do ambiente é inerente ao processo educativo, o item mais relevante a ser avaliado é o critério didático-pedagógico do software tendo em vista que qualquer desenvolvimento de um produto para educação é delineado pela concepção de aquisição do conhecimento, isto é, de que maneira o sujeito internaliza a aprendizagem (SCHLEMMER; FAGUNDES, 2001; SCHLEMMER, 2005). 
Na seção a seguir apresentam-se os procedimentos metodológicos.

\section{METODOLOGIA}

A abordagem desta pesquisa é qualitativa posto que investiga atributos funcionais que podem contribuir com a modelagem de um AVA quanto à usabilidade, interação, colaboração e aprendizagem para o ensino presencial do IFC - Campus Camboriú, a partir da análise das funcionalidades do Moodle já utilizado pela referida Instituição de Ensino no curso EAD PROEJA.

Quanto aos fins, tem-se uma pesquisa descritiva, que conforme GIL (2010) descreve as características de determinada população ou fenômeno, ou estabelece relações entre variáveis. Quanto aos meios de investigação optou-se pela pesquisa bibliográfica, documental e pelo estudo de caso.

$\mathrm{O}$ universo desta pesquisa foi dividido em três grupos escolhidos com base no estudo de Schlemmer e Fagundes (2001), considerando o paradigma interacionista/construtivista no qual a estruturação, planejamento e avaliação de um AVA deve ser conduzido de maneira interdisciplinar constituída por usuários, educadores, pedagogos, equipe técnica e gestores.

O Grupo 1 é composto pelo coordenador de suporte de EAD, pelos 3 (três) profissionais de TI do IFC, e também por 2 (dois) profissionais da SETIC da UFSC, responsáveis pelo Moodle UFSC, qualificados e experientes na instalação, desenvolvimento e manutenção do ambiente, tanto na modalidade a distância quanto na presencial.

O Grupo 2 é constituído pela equipe pedagógica do Curso, isto é por 1 (um) Coordenador de curso, 5 (cinco) professores e 4 (quatro) tutores.

O Grupo 3 é composto por 29 (vinte e nove) discentes concluintes do Curso de PósGraduação EAD em PROEJA.

Para esta pesquisa optou-se por entrevistas semi-estruturadas junto aos Grupos $1 \mathrm{e}$ 2 e questionário com o Grupo 3.

Foram elaborados dois roteiros de entrevista diferenciados, sendo um orientado ao Grupo 1 (Coordenador de suporte à EAD e Profissionais de TI), (Profissionais da SETIC, da UFSC) compostos por 18 (Dezoito) perguntas direcionadas à área técnica e, outro, para o Grupo 2 (Coordenador do curso de Pós-graduação EAD Proeja, professores e tutores, com 17 (Dezessete) questões voltadas para a área educacional e pedagógica.

O questionário constituído de 23 (vinte e três) questões foi enviado pelo correio eletrônico institucional da pesquisadora, por meio de um formulário personalizado pelo Google forms, aos 29 (vinte e nove) discentes que concluíram o curso de pós-graduação EAD em PROEJA.

Este formulário tinha o objetivo de identificar pontos fortes e fracos do Moodle quanto à sua usabilidade, interação, colaboração e aprendizagem e, verificar as ferramentas consideradas essenciais para otimizar o processo de aprendizagem no AVA. Dos 29 e-mails enviados, retornaram 15 questionários (cerca de 51\%.).

Na próxima seção são apresentados os resultados deste estudo.

\section{ATRIBUTOS FUNCIONAIS QUE PODEM CONTRIBUIR COM O DESENVOLVIMENTO DA MODELAGEM DO MOODLE PARA O ENSINO PRESENCIAL DO IFC - CAMPUS CAMBORIÚ}

Esta pesquisa identificou atributos funcionais por meio da análise das categorias usabilidade, interação, colaboração e aprendizagem com o objetivo de propor a 
modelagem de um AVA para o ensino presencial do IFC - Campus Camboriú, a partir dos seguintes resultados: principais ferramentas do Moodle utilizadas pelos professores, tutores e discentes do Curso de Pós-Graduação EAD PROEJA do IFC Campus Camboriú; pontos fortes e fracos verificados na plataforma Moodle pelos referidos sujeitos de pesquisa e também, pelos profissionais de TI da SETIC, da UFSC.

\subsection{Usabilidade}

Conforme pesquisa realizada junto aos Grupos 1, 2 e 3, para que o Moodle seja modelado para o ensino presencial do IFC - Campus Camboriú quanto à categoria "usabilidade", propõe-se que sejam analisadas as seguintes características: modularidade do software, design do layout, versão atualizada, critério para escolha das ferramentas, feedback aos usuários, integração dos módulos do Moodle aos demais sistemas utilizados pela Instituição, instalação, mantenimento e desenvolvimento do software na própria Instituição que o utiliza, capacitação de professores, servidores e alunos e avaliação contínua do AVA.

A modularidade é um dos pontos fortes da plataforma Moodle posto que o software é customizável e extensível, permitindo que se inicie uma instalação básica e, aos poucos, se acrescente e modifique recursos à medida que forem necessários. O Moodle apresenta características de um ambiente completamente aberto onde há possibilidades de agregar novas funcionalidades.

Quanto ao Layout sugere-se que seja adotado o "Design responsivo", de modo que o usuário seja capaz de acessar a plataforma Moodle a partir de qualquer dispositivo com acesso à Internet. Entretanto, é importante atentar para a configuração da interface, partindo da premissa que esta foi uma das reclamações durante a pesquisa. Sendo assim, as informações e recursos devem ser configuradas e apresentadas de maneira lógica, intuitiva e organizada, permitindo que os usuários intuam com clareza e sem maiores dificuldades quando forem acessar os módulos, as ferramentas, recursos e links.

A versão do Moodle é um requisito que, também, deve ser verificado e ponderado antes da instalação. E mesmo quando o software já está instalado é importante que a Instituição que o provê acompanhe a atualização das versões, considerando-se que a última versão é sempre a mais estável e segura. A vantagem de atualizar o Moodle está no fato de atualizar o núcleo e o módulo ser independente desta atualização. Desse modo, a atualização proporciona os seguintes benefícios, a saber: solução de problemas de segurança, incorporação de novas funcionalidades, mudanças na interface, dentre outros.

O critério para escolha das ferramentas é relevante para a modelagem de um AVA, pois deve-se verificar o impacto da instalação das ferramentas no Moodle, de modo que não coloquem em risco todo o ambiente. Em outras palavras, podem haver ferramentas mal desenvolvidas, fora dos padrões e a execução de uma atividade no ambiente pode inviabilizar o uso do Moodle.

Neste quesito observa-se a importância de ser constituída uma equipe multidisciplinar composta por profissionais de TI, Designer instrucional, Professores e Pedagogos, de modo a avaliar os objetivos do curso a ser ofertado e, também, ferramentas necessárias para atingir às expectativas na plataforma Moodle.

As ferramentas disponibilizadas pelo Moodle podem ser ofertadas em cursos a distância ou presencial. Todas as ferramentas podem influenciar no processo de aprendizagem, dependendo do modo como são utilizadas dentro de uma perspectiva educativa e, também, das necessidades apresentadas pelo professor, aluno, disciplina e curso. 
Sendo assim, sugere-se que sejam disponibilizadas todas ferramentas que o Moodle oferece quando da sua instalação, além de outros recursos como o módulo registro de presença e se necessário, o Moodle Provas, para que os alunos possam realizar as avaliações nos laboratórios da Instituição. E que as atividades e tarefas sejam configuradas de modo a fornecer o feedback aos usuários, possibilitando que os mesmos verifiquem o seu desempenho ao fim de cada utilização.

A colaboração com a Comunidade internacional do Moodle auxilia na usabilidade do Software quanto às falhas e às correções. É importante que a instituição que mantém o Moodle busque alternativas na referida comunidade e também as encaminhe, fomentando uma rede colaborativa de possibilidades e soluções conforme observado em diversas Instituições que utilizam o software.

A integração dos módulos do Moodle aos demais sistemas da Instituição é um excelente requisito a ser analisado, posto que permitirá que os dois sistemas conversem entre si e facilitem o uso e o acesso às informações.

Outrossim, é necessário que seja disponibilizada uma capacitação sobre o uso do Moodle para professores, servidores e alunos. Em especial, para professores, de modo que sejam auxiliados a realizar a montagem do material didático.

E por último, realizar constantemente a avaliação do Moodle no intuito de verificar os pontos positivos e negativos apresentados pelo software a partir da interação dos seus participantes, da aprendizagem, da colaboração e compartilhamento de informações, atividades e conhecimentos e, essencialmente, quando a avaliação abrange a utilização do software pelos discentes (SCHLEMMER; SACCOL; GARRIDO, 2006, 2007; OLIVEIRA; COSTA; MOREIRA, 2001).

Diante do exposto, sugere-se que seja utilizado o modelo interacionista/construtivista proposto por Schlemmer e Fagundes (2001) que analisa as perspectivas tecnológica, comunicacional-social, pedagógica e de gestão, considerando ainda, a sua aplicação por equipes interdisciplinares, constituídas por usuários, educadores, pedagogos, equipe de TI e gestores.

\subsection{Interação}

Com base nesta pesquisa, percebe-se que os profissionais (Professores e Tutores) devem dar mais atenção à questão do trabalho interativo no Moodle, fomentando por meio de atividades e tarefas a construção de um ambiente de aprendizagem participativo, flexível, centrado na interação, na troca de informação, experiências, conhecimento, desenvolvimento de atividades colaborativas, resolução de problemas e soluções de aprendizagem.

Nesta perspectiva, propõe-se que sejam ofertadas ferramentas que corroborem com o desenvolvimento de atividades e tarefas que favoreçam a interação em grupos assim como, atividades que permitam a expressão de opiniões, entrega e postagem de trabalhos, discussões entre os colegas de turma e profissionais envolvidos, a saber:correio eletrônico, fóruns de discussão, mural, portfólio, bate-papo e conferência.

\subsection{Colaboração}

Em relação à categoria "Colaboração", os atributos que podem contribuir com a modelagem do Moodle do ensino presencial do IFC - Campus Camboriú estão presentes em um ambiente participativo, centrado no trabalho colaborativo e em ferramentas que fomentem a colaboração individual e coletiva tais como, Fórum, E-mail, Mensagem, Conferência, Wiki e Compartilhamento de recursos. 
O ambiente Moodle foi pensado e desenvolvido para ser colaborativo. É a partir da interação entre alunos e professores que se constrói a colaboração. Nesse sentido, o modelo pedagógico adotado no curso ou na disciplina de um curso, a maneira como as ferramentas são utilizadas e como as atividades são conduzidas, corroboram efetivamente para uma aprendizagem colaborativa.

No quadro 1 seguem as ferramentas que podem fomentar a interação e colaboração no Moodle:

Quadro 1 - Ferramentas de interação e colaboração

\begin{tabular}{|l|l|}
\multicolumn{1}{|c|}{ Ferramentas } & \multicolumn{1}{c|}{ Quadro 1 - Ferramentas de interação e colaboração } \\
\hline Fórum & Organiza as discussões por assunto, por disciplina, por curso, por turma, por grupo. \\
\hline E-Mail/Mensagem & Ferramenta indicada para enviar e receber arquivos esclarecendo dúvidas, sugestões, etc. \\
\hline Conferência & $\begin{array}{l}\text { Ferramenta que realiza conferências em tempo real ou grupos de discussão que possibilitam aos } \\
\text { usuários o engajamento em uma troca colaborativa sobre tópicos do curso, disciplina, etc. }\end{array}$ \\
\hline Wiki & $\begin{array}{l}\text { Software que permite aos usuários editarem o conteúdo das páginas web, e também realizarem } \\
\text { discussões temáticas, reflexões, sínteses e construção do conhecimento. }\end{array}$ \\
\hline $\begin{array}{l}\text { Compartilhamento } \\
\text { de recursos }\end{array}$ & $\begin{array}{l}\text { Ferramenta que permite que um ou mais usuários compartilhem, arquivos, documentos, recursos de } \\
\text { seus computadores, etc. }\end{array}$ \\
\hline
\end{tabular}

Fonte: Schlemmer; Saccol; Garrido (2007); Moore; kearsley (2013); Peters (2012).

\subsection{Aprendizagem}

As ferramentas devem estar adequadas aos objetivos do curso e proporem o fomento da aquisição do conhecimento e também, contribuírem na avaliação da aprendizagem. Nesta categoria, sugere-se a análise de características que beneficiem o processo de ensino e aprendizagem no Moodle, quais sejam: Fórum, Mensagem, Conteúdos postados pelos professores, Exercícios, Livro Texto, Pastas com arquivos das disciplinas, Vídeos e Link de uma página Web.

Apresenta-se no quadro 2 atributos funcionais com propósitos de aprendizagem a serem ser modelados para o ensino presencial do IFC - Campus Camboriú:

\begin{tabular}{|c|c|c|}
\hline \multicolumn{3}{|c|}{ Quadro 2 - Atributos funcionais quanto à categoria "Aprendizagem" } \\
\hline Atributos funcionais & Descrição & Ferramentas \\
\hline Espaço de instrução & $\begin{array}{l}\text { Planeja o conteúdo a ser ensinado de uma maneira didática, } \\
\text { multimodal, plural e diversa, nas formas de representação } \\
\text { provenientes das multimídias. }\end{array}$ & Moodle \\
\hline $\begin{array}{l}\text { Informação } \\
\text { documentação }\end{array}$ & $\begin{array}{l}\text { Repositório e ordenação sistemática de informação e } \\
\text { documentação tematicamente relevantes, com um } \\
\text { gerenciamento eficaz e eficiente da informação. }\end{array}$ & $\begin{array}{l}\text { Glossário; Repositório de } \\
\text { documentos e de informação; } \\
\text { Biblioteca online }\end{array}$ \\
\hline Comunicação & $\begin{array}{l}\text { Apresentam ferramentas que permitem a comunicação } \\
\text { entre os usuários de um curso e a visibilidade dos trabalhos } \\
\text { desenvolvidos. }\end{array}$ & $\begin{array}{l}\text { Correio eletrônico; Fórum; } \\
\text { Mural; Mensagem; Portfólio; } \\
\text { Conferência. }\end{array}$ \\
\hline Colaboração & $\begin{array}{l}\text { Permite práticas colaborativas, de interação, } \\
\text { proporcionando o diálogo, a resolução de problemas e a } \\
\text { construção do conhecimento coletivamente. }\end{array}$ & Fórum; Wiki \\
\hline Hipertexto e hipermídia & $\begin{array}{l}\text { Programas lineares de aprendizagem apresentados por } \\
\text { hipertextos e hipermídia habilitam os alunos a } \\
\text { potencializarem os estilos de aprendizagem autônomos, } \\
\text { considerando, a aprendizagem individualizada, orientada } \\
\text { para resolução de problemas, e apoiam os processos } \\
\text { construtivos da aprendizagem e flexibilidade cognitiva. }\end{array}$ & Wiki \\
\hline Multimídia & $\begin{array}{l}\text { Conteúdos didáticos que podem ser apresentados em } \\
\text { diversas linguagens. }\end{array}$ & $\begin{array}{l}\text { Textos; Imagens; Vídeos; } \\
\text { Áudios, Gráficos; Animações } \\
\text { bi e tridimensionais.. }\end{array}$ \\
\hline Simulação & $\begin{array}{l}\text { Nesta ferramenta os alunos podem entrar em contato com a } \\
\text { realidade simulada. }\end{array}$ & $\begin{array}{lcr}\begin{array}{l}\text { Espaços } \\
\text { bibliotecas, }\end{array} & \text { virtuais } & \text { de } \\
\text { universidades. } & & \text { e } \\
\end{array}$ \\
\hline Realidade virtual & $\begin{array}{l}\text { Permite que os alunos se comuniquem de maneira interativa } \\
\text { com os objetos e pessoas tridimensionais, nos espaços e } \\
\text { paisagens de realidade virtual, simulados } \\
\text { tridimensionalmente. }\end{array}$ & $\begin{array}{l}\text { Software de realidade virtual } \\
\text { adaptado para pessoas com } \\
\text { necessidades especiais. }\end{array}$ \\
\hline
\end{tabular}




\begin{tabular}{|l|l|l|l|}
\hline Exploração & $\begin{array}{l}\text { Ferramenta de busca, de exploração que amplia a } \\
\text { informação com base nos próprios interesses e } \\
\text { preferências, fomentando a aprendizagem autônoma }\end{array}$ & $\begin{array}{l}\text { Ferramenta de busca externa e } \\
\text { interna. }\end{array}$ \\
\hline
\end{tabular}

Fonte: Peters (2012); Maciel (2012); Gonzales (2005).

A partir dos propósitos de aprendizagem identificados no quadro 2, percebe-se que não é o ambiente virtual que define a educação; ele pode sugestionar o processo de ensinoaprendizagem, mas não o determina (MILL; FIDALGO, 2007; SANTOS, 2011; PETERS, 2012).

Nesse sentido, quanto ao fomento da aprendizagem e da aquisição do conhecimento, sugere-se que sejam utilizados os seguintes recursos no Moodle, a saber: Arquivo, Livro, Página, Pasta, URL e Rótulo. E quanto às atividades: Chat, Fórum, Escolha, Ferramenta externa, Glossário, Lição, Questionário, Tarefa e Wiki.

Quanto à avaliação no Moodle propõe-se que seja utilizado o módulo "Laboratório de avaliação", o qual permite avaliar a revisão, a coleta de dados, os colegas de trabalho e os alunos por meio de um formulário de avaliação multicritérios pré-definidos pelo professor e também, o módulo "Pesquisa de avaliação" que fornece uma série de instrumentos de avaliação que são úteis para verificar e estimular a aprendizagem em ambientes online.

As ferramentas de avaliação do Moodle podem colaborar com os objetivos propostos pelos professores para o ensino presencial bem como, teste de múltipla escolha ou provas dissertativas, programação de horário para disponibilização da avaliação dos alunos, controle de tempo de realização, correção automática, cálculo e publicação das médias, geração de estatísticas $e$ feedback automático aos alunos sobre o seu desempenho.

No quadro 3 seguem as ferramentas que podem fomentar a aprendizagem e a avaliação da aprendizagem no Moodle:

Quadro 3 - Ferramentas que fomentam a aprendizagem e a avaliação da aprendizagem no Moodle

\begin{tabular}{|l|l|}
\hline Aprendizagem & $\begin{array}{l}\text { a) arquivo; b) livro; c) página; d) pasta; e) URL, f) rótulo; g) chat; g) fórum; h) } \\
\text { escolha; i) ferramenta externa; j) glossário; k) lição; 1) questionário; m) tarefa; } n \text { ) wiki. }\end{array}$ \\
\hline Avaliação & $\begin{array}{l}\text { a) laboratório de avaliação; b) pesquisa de avaliação; c) teste de múltipla escolha; d) } \\
\text { provas dissertativas; e) programação de horário para disponibilização da avaliação; } \\
\text { f) controle de tempo de realização; g) correção automática; h) cálculo e publicação } \\
\text { das médias; i) geração de estatísticas;j) feedback automático do desempenho dos } \\
\text { alunos. }\end{array}$ \\
\hline
\end{tabular}

Fonte: Dados primários (2015).

\section{CONSIDERAÇÕES FINAIS}

Com base neste estudo, concluiu-se que os atributos funcionais identificados podem colaborar com a modelagem do AVA Moodle para o ensino presencial do IFC - Campus Camboriú, de modo a desenvolver propósitos de aprendizagem, uma comunicação mais eficiente e eficaz com a comunidade acadêmica do ensino presencial, por meio de ferramentas que potencializem a aprendizagem individual e coletiva legitimando a busca pela informação e a construção do conhecimento de maneira interativa e colaborativa.

Os atributos funcionais propostos quanto à usabilidade tem por objetivo ofertar um ambiente atrativo, com baixa taxa de erros aos seus usuários permitindo que os mesmos atinjam níveis de produtividade na realização de suas tarefas, além de promover a avaliação de usabilidade do software de modo a verificar o desempenho da interação entre o usuário e o moodle.

Quanto à interação identificou-se que Moodle deve apresentar ferramentas que otimizem o processo de comunicação e interação entre discentes e docentes, como por 
exemplo, Fórum, Mensagem, E-mail e Wiki; possibilitando que o ambiente se torne participativo e fomente a busca pela informação, pelo conhecimento e pela aprendizagem, tendo como mediadora a interação social.

Em relação à colaboração concluiu-se que é essencial que o Moodle seja participativo, ofertando atividades colaborativas que propiciem a interação, resolução de problemas, construção colaborativa do conhecimento e da aprendizagem, conferindo aos usuários, liberdade, autonomia e criatividade no processo de aprendizagem por meio das seguintes ferramentas, quais sejam: Fórum, o E-mail, a Mensagem, a Conferência, a Wiki e o Compartilhamento de recursos.

De acordo com a categoria "Aprendizagem" pode-se dizer que é necessário que sejam utilizadas tanto ferramentas que fomentem a aquisição do conhecimento, quanto as que contribuam no processo de avaliação da aprendizagem, a saber: Arquivo, Livro, Página, Pasta, URL e Rótulo. E quanto às atividades: Chat, Fórum, Escolha, Ferramenta externa, Glossário, Lição, Questionário, Tarefa e Wiki.

Quanto às ferramentas de avaliação próprias para cursos de ensino presencial, somente o professor estará apto a avaliar e escolher as ferramentas necessárias para avaliar seus alunos e sua disciplina.

Percebeu-se, também, a necessidade de instituir tanto uma equipe de TI para gerir e desenvolver tecnologicamente melhorias no Moodle, quanto uma equipe multidisciplinar composta de professores, pedagogos, designer e profissionais de TI para avaliar a plataforma e propor melhorias quanto à parte técnica, pedagógica, comunicacional e de gestão do software.

A necessidade de institucionalizar, manter e desenvolver o Moodle para dar suporte às demandas tecnológicas e pedagógicas do ensino presencial foi uma realidade verificada.

Por fim, esta pesquisa buscou contribuir com a modelagem de um ambiente virtual de aprendizagem para o ensino presencial do IFC - Campus Camboriú, demonstrando que um AVA numa instituição de ensino, especialmente, no contexto do IFC pode exercer um papel diferenciador e agregador no processo de aprendizagem por meio da construção do conhecimento interativo e colaborativo.

\section{REFERÊNCIAS}

COLL, César; MONEREO, Carles. Psicologia da educação virtual: aprender e ensinar com as tecnologias da informação e da comunicação. Porto Alegre: Artmed, 2010.

GIL, Antônio Carlos. Métodos e técnicas de pesquisa social. 6. ed. São Paulo: Atlas, 2010.

GONZALES, Mathias. Fundamentos da Tutoria em Educação a Distância. São Paulo: Editora Avercamp, 2005.

HAGUENAUER, Cristina Jasbinschek; LIMA, Luciana Guimarães Rodrigues; CORDEIRO FILHO, Francisco. Comunicação e interação em ambientes virtuais de aprendizagem. In: CONGRESSO INTERNACONAL DE EDUCAÇÃO A DISTÂNCIA, 16., 2010, Foz do Iguaçu. Anais... Foz do Iguaçu: ABED, 2010. Disponível em: <http://www.abed.org.br/congresso2010/cd/252010213152.pdf >. Acesso em: 13 set. 2017. 
MACIEL, Cristiano (Org.). Ambientes virtuais de aprendizagem. Cuiabá: UFMT, 2012.

MILL, D.; FIDALGO F. Espaço, tempo e tecnologia no trabalho pedagógico: redimensionamento na Idade Mídia. Revista Brasileira de Estudos Pedagógicos, v. 88, n. 220, 2007.

MOORE, Michael G.; KEARSLEY, Greg. Educação a distância: sistemas de aprendizagem online. São Paulo: Cengage Learning, 2014.

OLIVEIRA, Celina Couto de; COSTA, José Wilson da; MOREIRA, Mercia. Ambientes informatizados de aprendizagem: produção e avaliação de software educativo. Campinas: papirus, 2001.

PETERS, Otto. A educação a distância em transição. São Leopolodo: Unisinos, 2012. SANTOS, E. Cibercultura: o que muda na Educação. Salto para o Futuro/Tv escola. Ano XXI, Boletim 3, 2011.

RIBEIRO, Fernanda Borges Vaz. Atributos funcionais que contribuem com o desenvolvimento da modelagem do ambiente virtual de aprendizagem Moodle para o ensino presencial: Instituto Federal Catarinense - Campus Camboriú. 2016. Dissertação (Mestrado Profissional) - Programa de Pós-Graduação em Administração Universitária, Universidade Federal de Santa Catarina, Santa Catarina, 2016. Disponível em:

https://repositorio.ufsc.br/bitstream/handle/123456789/171715/342975.pdf?sequence=1 \&isAllowed=y >. Acesso em: 30 set. 2017.

SCHLEMMER. Eliane. Metodologias para a educação a distância no contexto da formação de comunidades virtuais de aprendizagem. In: BARBOSA, Rommel Melgaço (Org.). Ambientes virtuais de aprendizagem. Porto Alegre: Artmed, 2005.

SCHLEMMER, Eliane; FAGUNDES, Léa da Cruz. Uma proposta para a avaliação de ambientes virtuais de aprendizagem na sociedade em rede. Informática na educação: teoria e prática, Porto Alegre, v.4, n.2, p. 25-36, dez. 2001.

SCHLEMMER, Eliane; SACCOL, Amarolinda; GARRIDO, Susane. Avaliação de ambientes virtuais de aprendizagem na perspectiva da complexidade. 2006. Disponível em: $<$ http://ltcead.nutes.ufrj.br/constructore/objetos/avaliacao\%20de\%20AVAs\%20na\%20perspectiva $\% 20 \mathrm{da} \% 20$ complexidade.pdf $>$. Acesso em: 03 set. 2017.

.Um modelo sistêmico de avaliação de softwares para educação a distância como apoio à gestão de ead. Revista de Gestão USP, São Paulo, v. 14, n. 1, p. 77-91, jan./mar. 2007. 